\title{
Formação de classes funcionais de estímulos musicais
}

\author{
Alex Roberto Machado \\ Elizeu Batista Borloti ${ }^{1}$ \\ Universidade Federal do Espírito Santo, Vitória-ES, Brasil
}

\begin{abstract}
Resumo: O objetivo deste experimento foi verificar o efeito do treino discriminativo sobre a formação de classes funcionais de melodias em andamentos e modos diferentes. Nove estudantes universitários do $2^{\circ}$ período da graduação em Psicologia foram divididos em três grupos: Modo, Andamento ou Misto. O software SomPsi, produzido especialmente para este estudo, treinou os participantes e gerou relatórios com informações sobre os desempenhos, nas quatro fases do procedimento. O treino proposto mostrou-se eficiente para a formação das classes funcionais de estímulos sonoros musicais. Observou-se que agrupamentos de estímulos pela propriedade andamento foram mais facilmente discriminados que os agrupados pela melodia. A manipulação combinada das duas propriedades, em condição convergente, permitiu desempenho superior dos participantes. A rápida formação de classes funcionais e equivalentes de estímulos musicais, a partir de procedimentos de ensino automatizado de relações condicionais entre estímulos musicais, indica a possibilidade de uso desse procedimento na iniciação à música.
\end{abstract}

Palavras-chave: música (psicologia), psicologia experimental, análise do comportamento.

\section{Formation of functional classes of musical stimuli}

\begin{abstract}
This experiment aimed to verify the effect of discriminative training on the formation of functional classes of melodies, with different modes and tempos. Nine students attending the 2nd period of undergraduate psychology program were divided in three groups: Mode, Tempo and Mixed. The software SomPsi, specifically designed for this study, trained the participants and generated reports with information about performances in the four stages of the procedure. The proposed training was efficient for the formation of functional classes of sound stimuli. We observed that stimuli grouped by tempo were more easily discriminated than those grouped by melody. Combined manipulation of the two proprieties, in convergent condition, permitted participants' superior performance. The rapid formation of functional classes and equivalent musical stimuli, as from automated teaching of conditional musical stimuli relations, indicates the possibility of using this procedure in introduction to music.
\end{abstract}

Keywords: music (psychology), experimental psychology, behavioral assessment.

\section{Formación de clases funcionales de estímulos musicales}

Resumen: El objetivo de este experimento fue verificar el efecto del entrenamiento discriminatorio sobre la formación de clases funcionales de melodías en diversos modos y andamientos. Nueve estudiantes del segundo año de graduación en psicología, divididos en tres grupos: Modo, Andamiento y Mixta. El software SomPsi, producido específicamente para este estudio entrenó a los participantes y generó relatos con informaciones sobre las actuaciones en las cuatro fases del procedimiento. El entrenamiento propuesto se mostró eficiente para la formación de las clases funcionales de estímulos sonoros musicales. Se observó que la agrupación de estímulos por la propiedad tiempo fue más fácilmente discriminada que los agrupados por la melodía. La combinación de la manipulación de las dos propiedades en condición convergente, tiene un rendimiento superior de los participantes. La rápida formación de clases funcionales y de los estímulos musicales equivalentes de procedimientos automatizados para la enseñanza de condicionar las relaciones entre los estímulos musicales, indica la posibilidad de utilizar este procedimiento en la introducción a la música.

Palabras clave: música (psicología), psicología experimental, análisis de la conducta.

O que é Música? Muniz e Castro (2005, p. 706), em seu dicionário, descrevem-na em um verbete como " 1 . Arte de combinar sons e coordenar fenômenos acústicos de maneira agradável ao ouvido. 2. Qualquer composição musical. 3. Execução de peça musical. 4. Música escrita; solfa. 5. Conjunto de músicos. 6. Conjunto de sons harmoniosos; orquestra".

As definições acima parecem tratar a música como ação de alguém e como propriedade de estímulos ambientais

\footnotetext{
1 Endereço para correspondência:

Prof. Dr. Elizeu Batista Borloti. Universidade Federal do Espírito Santo. Centro de Ciências Humanas e Naturais. Programa de Pósgraduação em Psicologia. Departamento de Psicologia Social e do Desenvolvimento. Av. Fernando Ferrari, 514, Campus Universitário de Goiabeiras/UFES, CEMUNI VI. CEP 29075-910. Vitória-ES, Brasil. E-mail:elizeu@comporaec.com.br
}

produzidos por essa ação. Na primeira definição, ainda que a palavra "arte" tenha sido colocada, os verbos utilizados dão caráter de ação do músico, que pode ser corroborada pela terceira definição. No entanto, a sexta definição dá maior ênfase à propriedade "harmonia" de um "conjunto de sons", independente do agente que o produziu. Isto sugere, no mínimo, duas funções assumidas pela música: evento comportamental ou ambiental.

Na Análise do Comportamento há investigações empíricas usando estímulos sonoros, a maioria delas (descritas na subseção intitulada "função reforçadora") utilizou estímulos sonoros como eventos ambientais do tipo estímulos discriminativos (SD's). Entretanto, os estímulos sonoros mais frequentemente usados foram sons uniformes (Pierrel \& Sherman, 1960; Maltzman, Holz, \& Kunze, 1965; Stebbins, 1966; Schusterman, Balliet, \& Nixon, 1972; Segal \& 
Harrison, 1978; Lewis \& Stoyak, 1979; Harrison, 1988), de voz humana (Reed, Howell, Sackin, Pizzimenti, \& Rosen, 2003) ou de campainhas/clicks (Crites, Harris, Rosenquist, \& Thomas, 1967; Farris, 1967). Diferente destas modalidades de estímulos sonoros, o presente estudo utilizou estímulos sonoros musicais complexos (melodias) em contexto de pesquisa básica, apresentando um delineamento que visou adicionar dados à discussão conceitual sobre o agrupamento de estímulos em classes. Portanto, este artigo descreve um experimento cujo objetivo foi verificar o efeito do treino discriminativo sobre a formação de classes funcionais de melodias em andamentos e modos diferentes.

Para o alcance deste objetivo, apresenta-se, a seguir, uma revisão bibliográfica dos estudos que utilizaram a música como evento comportamental e como evento ambiental, bem como dos estudos sobre a formação de classes de estímulos. Depois, apresenta-se o método, resultados e conclusão.

\section{Música como evento comportamental}

O comportamento que produz música pode ser analisado como os demais comportamentos operantes. Mesmo que tal produto seja admirado como arte, a aquisição/manutenção de comportamentos de manuseio ordenado de estruturas anátomo-fisiológicas e/ou instrumentos musicais depende da função de tais atividades num dado contexto cultural. Considerados os sons vocalizados emitidos pela movimentação das cordas vocais como verbais (Skinner, 1957), os sons não vocalizados produzidos por essa parte e os produzidos por outras partes do corpo, por intermédio ou não de instrumentos, e com a função de produzir determinado efeito em uma audiência - especialmente treinada por uma cultura musical - seriam também verbais. De fato, Skinner (1957, p. 29) lembra que, na definição de comportamento verbal como comportamento reforçado por intermédio de outras pessoas, não é especificada qualquer forma, modo ou meio de emissão da resposta, e nem o seria possível fazer, pois “qualquer movimento capaz de afetar outro organismo pode ser verbal". Entretanto, a ênfase no comportamento vocal seria em função de esse ser mais comum e "quase necessariamente verbal". É por isto que a ênfase nos verbais vocais se mantém ao longo do livro do autor.

Frequentemente, o produto do comportamento musical (a música produzida) funciona como um evento ambiental, tanto para os ouvintes quanto para o próprio músico. Talvez, por esta razão, foram encontrados, na Análise do Comportamento, mais trabalhos que estudaram a música como um estímulo (a serem descritos na subseção intitulada "função reforçadora") do que trabalhos que estudaram o comportamento musical per se (Collins, 2005; Ebie, 2004; McPherson, 2005; Sadakata, Ohgushi, \& Desain, 2004). São raros os estudos desse repertório e da sua relação com outros eventos ambientais e comportamentais.

\section{Música como evento ambiental}

Como evento ambiental, a música pode ser definida por alterações de estímulos sonoros combinados (pela manipulação de um humano com a função de produzir determinado efeito em uma audiência) que ocorrem no ambiente, antecedendo ou sendo consequência de um determinado evento comportamental, de modo que controlam a ocorrência deste último. Neste artigo, a música será estudada na sua característica de ser um produto do comportamento, evitando-se a qualificação dessa combinação de estímulos sonoros como "harmoniosa" ou "agradável", comum a algumas definições abrangentes, tais como as do dicionário supracitado. Deste ponto em diante, os termos música e estímulos sonoros musicais serão tratados como sinônimos. As funções dos estímulos musicais são descritas a seguir.

\section{Função eliciadora}

Os estímulos sonoros podem eliciar respostas reflexas. Apesar de alguns estímulos sonoros serem incondicionados, o consenso é que estímulos sonoros eliciadores adquiriram esta função pelo fato de terem sido "seguidos ou 'reforçados' por um estímulo efetivo, frequentemente chamado de estímulo eliciador incondicionado" (Skinner, 1953, p. 53), tornando-se estímulos condicionados. É assim que uma música torna-se eliciadora de medo, prazer sexual ou qualquer outra forma de emoção. De fato, "os efeitos emocionais da música (...) são largamente condicionados" (Skinner, 1953, p. 57).

Embora tenha apresentado algumas conjecturas sobre as funções de estímulos musicais de eliciar respondentes, como a supracitada, Skinner não ilustrou tais funções com pesquisas básicas ou aplicadas. De fato, alguns exemplos de aplicação da música como estímulo eliciador estão em estudos conduzidos por pesquisadores não analistas do comportamento, em trabalhos com musicoterapia (Chlan, 1998; Hanser \& Thompson, 1994; Hatem, Lira, \& Mattos, 2006; Hoskyns, 1988; Koger, Chapin, \& Brotons, 1999; Macdonald e cols., 2003; Nilsson, Unosson, \& Rawal, 2005; Odell, 1988; Todres, 2006; White, 1992) e comportamento do consumidor (North \& Hargreaves, 1999; North, Shilcock, \& Hargreaves, 2003; Wilson, 2003).

\section{Função reforçadora}

Com esta função, os estímulos sonoros são consequências de determinada resposta e produzem o aumento da sua probabilidade de emissão no futuro. Entre os estudos que utilizaram a música como reforçador, há um grupo que objetivou mudar certos comportamentos tornando-os "adaptados" ou "produtivos" (Barmann, Croyle-Barmann, \& Mclain, 1980; Wilson \& Hopkins, 1973; Greene, Bailey, \& Barber, 1981; Lowe, Beasty, \& Bentall, 1983; Hume \& Crossman, 1992). Um segundo grupo ocupou-se de comparar também o valor reforçador de alguns outros estímulos juntamente com os musicais (Ferrari \& Harris, 1981; Thompson, Heistad, \& Palermo, 1963; Vollmer \& Iwata, 1991; Kennedy \& Souza, 1995). 
Há, ainda, estudos que empregaram controle aversivo, utilizando estímulos sonoros como reforçadores negativos (Kelly, 1980; Mcadie, Foster, \& Temple, 1996; Harrison \& Abelson, 1959; Kelly, 1980; McAdie e cols., 1996). Nesses trabalhos, a remoção de um estímulo sonoro aversivo como consequência da resposta foi eficiente para aumentar a sua taxa de emissão.

\section{Função discriminativa}

Neste caso, o estímulo sonoro, com função de estímulo discriminativo, estabelece a ocasião para que um organismo emita uma resposta que é seguida por um reforçador positivo (Lundin, 1977). Esta função reúne a maior parte dos estudos usando estímulos sonoros na Análise do Comportamento (Beecher \& Harrison, 1971; Burlile, Feldman, Craig, \& Harrison, 1985; Crites, Harris, Rosenquist, \& Thomas, 1967; Downey \& Harrison, 1972, 1975; Farris, 1967; Green, 1975; Harrison \& Briggs, 1977; Harrison, Iversen, \& Pratt, 1977; Harrison, 1988; Herman \& Gordon, 1974; Lewis \& Stoyak, 1979; Neill \& Harrison, 1987; Neill, Liu, Mikati, \& Holmes, 2005; Raslear, 1975; Raslear, Pierrel-Sorrentino, \& Brissey, 1975; Reed, Howell, Sackin, Pizzimenti, \& Rosen, 2003; Schusterman, Balliet, \& Nixon, 1972; Segal \& Harrison, 1978; Stebbins, 1966). Em geral, esses estudos assemelham-se: frequentemente utilizaram ratos como sujeitos e sons simples como estímulos (descritos pela frequência em Hertz). A maioria foi publicada na década de 1970, sendo poucos na segunda metade da década de 1960 e outros poucos na segunda metade dos anos 1980. Entre os mais recentes, cita-se o de Reed e cols. (2003), que teve como objetivo investigar a habilidade de ratos discriminarem um som vocal dentre vários sons vocais. Apenas um rato (de uma amostra de dez) não aprendeu a discriminar entre os estímulos.

\section{Função condicional e função estabelecedora}

Há, ainda, estudos que utilizaram os estímulos sonoros como contexto para que determinado estímulo adquirisse função de $\mathrm{SD}$ e, consequentemente, funcionassem como estímulos condicionais (Galizio, Stewart, \& Pilgrim, 2004; Hayes, Thompson, \& Hayes, 1989; Herman \& Gordon, 1974); há um estudo que os utilizaram como estímulo estabelecedor (North e cols., 2003). Descritas as funções dos estímulos musicais, a seção seguinte discute o agrupamento de estímulos em classes, visando à ampliação do controle que tal agrupamento permite.

\section{Agrupamentos de estímulos sonoros em classes}

A formação de classes pode ocorrer por relações entre estímulos, seja por similaridade física ou atributos comuns, por relações arbitrárias mediadas por resposta comum ou por relações arbitrárias (De Rose, 1993). A similaridade física ou atributos comuns diz respeito às propriedades físicas de classes de estímulos que controlam uma resposta comum. Como exemplo, há a classe de estímulos que controlam a resposta verbal "violões", formada por objetos com propriedades físicas comuns (corpo curvilíneo de madeira, cordas e timbre característico, entre outras).

As relações arbitrárias mediadas por resposta comum dizem respeito a relações entre estímulos que, mesmo sem similaridade física ou atributos comuns, arbitrariamente, ocasionam a ocorrência de uma resposta comum. De Rose (1993) afirma que os estímulos assim agrupados tornam-se "funcionalmente equivalentes", constituindo uma "classe funcional". Ainda, segundo De Rose (1993, p. 286), “esta classe só é demonstrada quando variáveis aplicadas diretamente sobre um estímulo têm efeito similar sobre os demais". Nas palavras de Sidman, Wynne, Maguire e Barnes (1989, p. 447), essa classe emerge "quando diferentes estímulos discriminativos em um set de contingências de três termos (em discriminações simples) ocasionam a mesma resposta". Assim, diante de uma sala com diferentes objetos, e com a instrução verbal "limpar os instrumentos musicais", o encarregado do serviço responde limpando apenas o violão e o piano, a despeito dos demais estímulos presentes que, naturalmente, não seriam instrumentos musicais. A resposta comum "utilizar-se de habilidades específicas para tocar alguma melodia", possível para ambos os estímulos, violão e piano, é mediadora nesta relação arbitrária, que os agrupa na classe denominada pela comunidade verbal de "instrumentos musicais". A resposta comum é dita "mediadora" por unir estímulos arbitrariamente, não por sua topografia, mas por sua função em determinada contingência. As topografias das respostas, entendidas aqui como as técnicas empregadas para tocar um piano ou um violão (respostas motoras de membros superiores e inferiores do corpo do instrumentista), diferem entre si. Contudo, a função-produto "uma melodia tocada" é possível às respostas em ambos estímulos, o que permite que sejam agrupados numa mesma classe.

O experimento de Vaughan (1988) tem sido citado (por exemplo, por Galvão, 1993) como o mais expressivo trabalho sobre a formação de classes funcionais de estímulos a partir do desempenho de animais, por demonstrar a formação das relações arbitrárias mediadas por resposta comum. De fato, foi a primeira demonstração inequívoca de que isto ocorre. Nesse experimento pombos foram ensinados a discriminar entre dois grupos de estímulos: aquele diante do qual uma mesma resposta emitida (bicar um disco) foi reforçada e aquele diante do qual nenhuma resposta emitida produziu reforçadores. $\mathrm{O}$ primeiro grupo foi chamado positivo $(\mathrm{S}+)$ e o segundo, negativo (S-).

Trabalhos mais recentes (Eikeseth \& Smith, 1992; Meehan, 1999; Stromer, Mackay, \& Remington, 1996; Tomonaga, 1999) trazem algumas alterações no método do estudo de Vaughan (1988). Wirth e Chase (2002), além de Goulart, Galvão e Barros (2003), por exemplo, lançaram mão do uso de reversões discriminativas com o intuito de analisar a estabilidade das classes funcionais formadas. Nesses trabalhos não foram utilizados estímulos musicais. 
De Rose (1993) avança na análise, ao citar um terceiro grupo de relações que poderiam formar classes de estímulos: as relações arbitrárias entre estímulos. Elas ocorrem sem o requisito explícito de uma resposta mediadora, mas com o estabelecimento da relação direta entre estímulos. Desta forma, não há a necessidade de que os estímulos controlem respostas com a mesma função. O ensino tradicional da música, a partir do pareamento de estímulos sonoros e instruções verbais que os nomeiam, utiliza tais relações. Não é a semelhança física, ou uma resposta comum que une determinado som a uma nomenclatura, por exemplo, Dó, Ré ou Mi. Na verdade, é reforçada a emissão da resposta verbal falada/escrita "Dó", diante de um estímulo sonoro particular, e não de outros.

Os estudos de Sidman e Tailby (1982) e Sidman (1992) contribuíram por oferecer uma especificação formal dos critérios para verificar a formação de classes de equivalência entre estímulos arbitrários. Os critérios simetria, transitividade e reflexividade foram estabelecidos a partir das propriedades das relações entre esses estímulos (De Rose, 1993).

Tais conceitos possibilitam questionar sobre as possíveis interfaces entre as classes funcionais e as classes equivalentes. Sidman e cols. (1989) apresentam a importância de se mostrar equivalência em classes funcionais, citando que: "ao se mostrar empiricamente que classes funcionais implicam relações equivalentes no comportamento, independente de suas diferentes definições e processos de testagem, nós alcançaríamos um notável grau de elegância teórica, previsibilidade empírica e integração potencial de dados" (Sidman e cols., 1989, p. 420).

Nesta direção, Sidman e cols. (1989) sugerem um procedimento em que, usando os estímulos A, B e C, são treinadas relações $\mathrm{AB}$ num primeiro momento, seguidas de treino de relações BC. Num terceiro momento, investiga-se se a relação $\mathrm{AC}$ se estabeleceu sem treino direto prévio. Duas mudanças foram realizadas nesse procedimento em relação aos demais em discriminações simples: (a) uso de três - ao invés de dois - estímulos por tentativa (um S+ e dois S-'s, o que permite, segundo o autor, a mudança da nomenclatura de reversão discriminativa para o termo mais genérico mudanças da contingência, visto que não seriam apenas dois grupos a serem discriminados); (b) a sinalização do $\mathrm{S}+$ aos sujeitos em uma das discriminações, já que na primeira tentativa, eles nunca teriam de experimentar a extinção antes de terem contato com uma mudança de contingência. Uma vez que classes funcionais tenham emergido, os sujeitos do estudo do autor deveriam ser capazes de passar por um completo bloco de teste sem erros.

Certos trabalhos foram influenciados por algumas das sugestões acima, apresentando avanços metodológicos (Goulart e cols., 2003; Schusterman \& Kastak, 1998). Jitsumori, Siemann, Lehr e Delius (2002), por exemplo, utilizaram pombos como sujeitos, treinando-os num procedimento de discriminação simples de dois grupos de estímulos (A1, B1, C1, D1 e A2, B2, C2, D2). Os pássaros foram expostos a uma série de reversões discriminativas para o estabelecimento das classes $\mathrm{AB}$ e $\mathrm{CD}$. Posteriormente, foram treinados na relação $\mathrm{AC}$ e BD para que, posteriormente, fosse testada a emergência das relações $\mathrm{AD}$ e $\mathrm{BD}$. Dois dos quatro pombos exibiram a emergência de uma dessas relações não treinadas, ou seja, relações de transitividade.

Já o trabalho de Tyndall, Roche e James (2004) consistiu num treino discriminativo com dois grupos (seis estímulos cada), seguido por um pareamento com o modelo. Esse pareamento foi testado em duas condições: utilizando-se somente estímulos do grupo $\mathrm{S}+$ como estímulos modelo e de comparação, e utilizando-se três estímulos de cada grupo - S+ e S- - como modelo e comparação. Os resultados mostraram que, em média, foram necessárias mais tentativas de teste para formar relações equivalentes quando os estímulos utilizados faziam parte de uma mesma classe funcional, do que quando eram de diferentes classes funcionais. Os autores justificam esse resultado pelo fato do treino de pareamento de estímulos exigir um repertório contrário ao previamente estabelecido, demandando a exclusão de estímulos da classe funcional pré-formada. $\mathrm{O}$ treino sinaliza o quão promissora pode ser a investigação das relações entre classes funcionais e equivalentes, bem como o quão rica é a possibilidade de uma variabilidade nos caminhos metodológicos para tal. Entretanto, os estudos que se propuseram a isto não utilizaram estímulos sonoros, apenas visuais.

\section{Método}

Este estudo pode ser classificado como simulado por analogia, prospectivo e transversal (Meltzoff, 2001). Segundo os objetivos, classifica-se como experimental, e quanto à natureza dos dados, quantitativo (Gonçalves, 2005). O delineamento utilizado foi o de sujeito como próprio controle, com uma configuração ABCA, sendo "A" fases de teste e "B" e "C", fases de intervenções.

\section{Participantes}

Foram organizadas três condições experimentais semelhantes, cada participante só poderia realizar uma delas. Visando-se maior riqueza de dados, optou-se por trabalhar com três participantes para cada condição experimental (Modo, Andamento ou Mista), totalizando nove participantes. Os critérios de inclusão na amostra foram: estar matriculado no $2^{\circ}$ período do curso de Psicologia da Faculdade Pitágoras, Linhares-ES, ter idade entre 18 e 22 anos e não ter histórico de treino formal/acadêmico em música. Embora nenhum teste audiométrico tenha sido utilizado, era esperado que alunos que participam regularmente de aulas expositivas orais não tivessem qualquer handicap auditivo importante, que os incapacitassem de realizar o procedimento. Os participantes assinaram o Termo de Consentimento Livre e Esclarecido (TCLE) no qual foram informados sobre a natureza da pesquisa, a possibilidade de encerrarem o procedimento em qualquer momento e a garantia de anonimato na ocasião de divulgação de dados da pesquisa. 


\section{Local, instrumentos e material}

A coleta de dados ocorreu no laboratório de informática da faculdade supracitada, com fones de ouvido pessoais. Para tal, o projeto da pesquisa recebeu aprovação da Comissão de Ética da instituição em que os dados foram coletados e do Comitê de Ética da Universidade Federal a qual os autores são filiados.

Baseado no estudo de Gagnon e Peretz (2003), foram utilizados 12 estímulos sonoros no total (cada condição experimental com seis estímulos). Os sons foram gerados a partir do software Cakewalk Sonar 6, utilizado em gravações musicais. Os estímulos apresentam o som MIDI Bright Acoustic Piano (com sonoridade semelhante a um piano acústico com timbre mais agudo), nas seguintes tonalidades: Dó maior, Dó menor, Mi maior, Mi menor, Láb maior e Láb menor, nos andamentos de $220 \mathrm{Bpm}$ (batimentos por minuto) em andamento rápido e $110 \mathrm{Bpm}$ (andamento lento). Os arquivos de áudio foram normalizados a $-16 \mathrm{db}$, e tinham 6 segundos de duração. A transposição de tonalidades de Dó, Mi e Láb se deu para que os estímulos tivessem a mesma distância de dois tons. As representações das melodias na partitura são mostradas na Figura 1.

Quanto às condições experimentais, essas foram organizadas em função dos estímulos, conforme descrito a seguir:

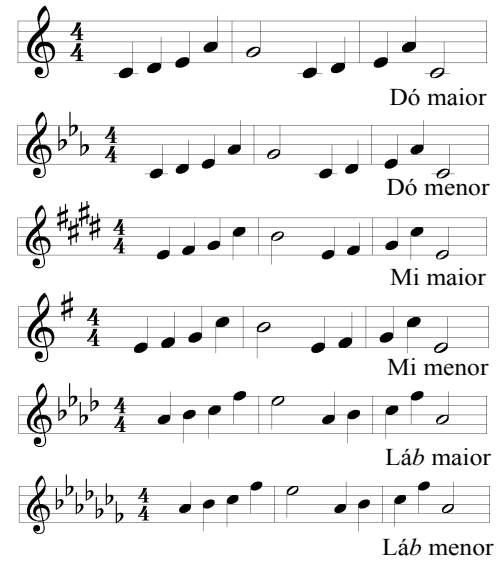

Figura 1. Notação em partitura das melodias musicais utilizadas no procedimento.

na condição Modo os estímulos apresentados tinham mesmo andamento e diferiam na tonalidade (maior/menor). Na condição Andamento todos os estímulos estavam na tonalidade maior, diferindo quanto à velocidade (rápido/lento). A condição Mista combinava diferenças de modo e andamento (Tabela 1). A fim de facilitar a identificação de cada estímulo em relação a cada condição experimental, eles foram nomeados como som1, som2, som3, som4, som5 e som6.

Tabela 1

Condições experimentais e propriedades dos estímulos sonoros

\begin{tabular}{lcccccc}
\hline \multirow{2}{*}{ Legenda } & \multicolumn{2}{c}{ Condição Modo } & \multicolumn{2}{c}{ Condição Andamento } & \multicolumn{2}{c}{ Condição Mista } \\
& Tonalidade & Andamento & Tonalidade & Andamento & Tonalidade & Andamento \\
\hline Som 1 & Dó maior & Rápido & Dó maior & Rápido & Dó maior & Rápido \\
Som 2 & Dó menor & Rápido & Dó maior & Lento & Dó menor & Lento \\
Som 3 & Mi maior & Rápido & Mi maior & Rápido & Mi maior & Rápido \\
Som 4 & Mi menor & Rápido & Mi maior & Lento & Mi menor & Lento \\
Som 5 & Láb maior & Rápido & Láb maior & Rápido & Láb maior & Rápido \\
Som 6 & Láb menor & Rápido & Láb maior & Lento & Láb menor & Lento \\
\hline
\end{tabular}

O desempenho esperado na condição Modo era a formação das classes de estímulos "melodias em modo maior" e "melodias em modo menor". Na condição Andamento, esperou-se a formação das classes "melodias em andamento rápido" e "melodias em andamento lento". Enfim, esperavase que os participantes formassem, na condição Mista, as classes "melodias em modo maior e andamento rápido" e "melodias em modo menor e andamento lento".

O procedimento foi dividido em quatro fases, sendo utilizado, para a coleta de dados, o software SomPsi. Apresentado como um jogo, o software executou todo o treinamento dos participantes e gerou relatórios com informações sobre desempenhos fase a fase. No programa, os participantes visualizavam a tela composta por um "Botão superior" que tinha a imagem de uma nota musical, o "Botão de escolha", arredondado, que o participante "escolheria" clicar ou não e o botão "Avançar". O desempenho esperado dos participantes
- em todas as fases - foi o de clicar no "Botão superior" para escutar o estímulo sonoro e em seguida, clicar ou não no "Botão de escolha". Para passar à tela seguinte, o participante deveria clicar no botão "Avançar". Tal configuração baseia-se no procedimento de Vaughan (1988), denominado de go - no go, em que é apresentado ao sujeito um determinado estímulo e, em seguida, oferecidas duas opções: (a) "escolher" o estímulo, emitindo uma resposta específica, ou (b) "desprezá-lo", não emitindo a resposta de "escolha". Na presente pesquisa, propôs-se: (a) que o botão superior oferecesse controle ao participante sobre o momento que ouviria o estímulo, ainda que pudesse ouvir cada estímulo uma única vez por tentativa; (b) que o participante emitisse a resposta de clique no "Botão de escolha" apenas diante de estímulos musicais de uma mesma classe, e (c) que encerrasse cada tentativa com o clique no botão "Avançar". Tais alterações buscavam manter a atenção do participante no procedimento como consequência de uma maior autonomia. 
As "relações arbitrárias mediadas por resposta comum" foram treinadas com transferência de função na mudança de fase. Entretanto, ao contrário de alguns trabalhos que se interessaram em gerar classes funcionais por repetidas reversões discriminativas, optou-se aqui por substituir várias reversões por apenas uma, da Fase2 para a 3. Na Fase 2, treinou-se a relação $\mathrm{AB}$; na Fase 3, a relação $\mathrm{BC}$. Isto, seguido pela verificação da emergência de relações não treinadas na última fase, aproxima este trabalho de procedimentos comuns em experimentos da categoria "relações arbitrárias entre estímulos".

\section{Procedimento de coleta de dados}

$\mathrm{Na}$ tela inicial do programa os participantes eram orientados a clicarem em uma condição experimental: A (melodia), $\mathrm{B}$ (ritmo) e $\mathrm{C}$ (mista). Apenas as letras referentes às condições apareciam, sem esclarecer sobre o significado de cada condição. A coleta de dados se deu para todos os participantes simultaneamente.

Todas as fases do programa iniciavam com uma tela com instruções escritas, seguidas pelo bloco de telas de treino discriminativo. A resposta de clique no botão de escolha era registrada para todas as telas, fase a fase.

Na Fase 1 - Linha de Base - as instruções informavam ao participante que o objetivo era treiná-lo no manejo do procedimento. Aqui foi medido o nível operante. Foram apresentados os seis estímulos sonoros que o participante teria contato nas fases seguintes. Tal apresentação ocorreu de forma randômica, por duas vezes, contabilizando um total de 12 telas. Após clicar no botão superior e ouvir o estímulo sonoro, o participante poderia clicar no botão de escolha e em seguida no botão "Avançar", ou clicar diretamente no botão "Avançar". A passagem de uma tela à outra dependeu unicamente do clique no botão "Avançar". O critério para avançar para a próxima etapa foi a apresentação das 12 telas.

Na Fase 2 - Treino Discriminativo - as instruções informavam ao participante que ele deveria descobrir para quais estímulos clicar no botão de escolha, clicando em seguida no botão "Avançar"; e para quais deveria clicar diretamente no botão "Avançar". O objetivo aqui foi ensinar a discriminação dos estímulos em classes clicando no botão de escolha, bem como medir a taxa de resposta de clique no botão de escolha para duas classes. Foram apresentados quatro estímulos sonoros (som1, som2, som3, som4), de forma randômica, por cinco vezes, contabilizando um bloco de 20 telas. As respostas de clicar no botão de escolha foram seguidas pela adição de 10 pontos (os contadores de pontos eram visíveis apenas nas fases 2 e 3 ) diante de apenas dois dos quatro estímulos (som1 e som3, aqui S+'s). O critério para passar desta fase do procedimento foi acertar 19 telas num mesmo bloco (clicar no botão de escolha somente nas telas que exibiram um som $\mathrm{S}+)$. Caso isto não ocorresse ao final do primeiro bloco (20 telas iniciais), um novo bloco de 20 telas seria apresentado, podendo o participante repetir a tentativa quantas vezes fossem necessárias para atingir o desempenho mínimo. O software registrava o número de repetições realizadas para cada fase, mas não as exibia aos participantes.
A Fase 3 - Reversão Discriminativa - era uma réplica da anterior quanto ao comportamento exigido e ao critério para ganhar pontos. Seu objetivo foi manter um repertório comum diante de estímulos de uma mesma classe, contudo, invertendo a resposta exigida diante de cada classe. Além desta, houve outras alterações: dois dos estímulos utilizados na Fase 2 foram retirados (som1 e som2) e os dois estímulos restantes (apresentados na linha de base, mas não na fase de treino discriminativo) foram adicionados (som5 e som6). Os estímulos remanescentes da fase anterior tiveram suas funções invertidas (se eram S+'s, passaram a ser S-'s). As funções dos estímulos adicionados acompanhavam a dos estímulos pares e ímpares remanescentes (som3 e som5 foram os S-'s; som4 e som6, os S+'s). Foi informado ao participante sobre o desempenho necessário (como feito na fase anterior), mas não sobre a mudança na função discriminativa das classes.

A Fase 4 - Teste - era uma réplica da Fase 1 (Linha de base) e teve como objetivo o levantamento de dados sobre o desempenho sem a apresentação de estímulos (pontos) como consequência. Visava-se a comparação entre os dados das fases 1 e 4 para avaliar a eficiência da intervenção nas fases intermediárias. Projetos-pilotos indicaram a necessidade de que as instruções para esta fase fossem diferentes das demais. Caso contrário, grande parte dos participantes continuava a se comportar como nas fases anteriores, ou seja, aguardavam as mensagens que informavam o acerto e total de pontos, enviesando o resultado. Com a mudança realizada, inclusive do layout visual (que se apresentou mais colorido e com uma organização do texto diferente do padrão utilizado para as instruções das demais fases), esperava-se que os participantes ficassem mais sobre o controle da regra descrita como a "instrução da fase".

\section{Resultados e Discussão}

Os resultados mostram diferenças no processo de aprendizagem para a formação das classes dos estímulos apresentados em cada condição experimental. A comparação dos dados referentes ao desempenho dos participantes na Fase 4 (teste) permite apontar a formação das classes de estímulos musicais.

O estudo de Hyde e Peretz (2004) sinaliza para o provável fato de que o repertório de discriminação de estímulos musicais de andamentos diferentes é mais fácil de ser aprendido do que o repertório discriminativo de tonalidades diferentes. De certa forma, o estudo de Bella, Peretz, Rousseau e Gosselin (2001) confirma tal afirmação, pois, ao comparar o desempenho de adultos e crianças ( 3 a 8 anos) na classificação de músicas nas categorias "alegre" ou "triste", identificou que as crianças mais jovens identificavam unicamente a propriedade andamento. Os resultados a seguir permitem discutir esta questão, uma vez que serão apresentados dados referentes à discriminação de modos e andamentos, tomados separadamente ou simultaneamente. Os dados de todos os participantes estão representados na Figura 2. Como a quantidade de apresentações de estímulos variava de acordo com 
a fase, optou-se por utilizar como medida a frequência relativa, dividindo o total de respostas de clique no botão de escolha pelo total de apresentações daquele estímulo. Assim, o participante que clicou no botão de escolha para todas as vezes que lhe foi apresentado o estímulo " $\mathrm{X}$ ” terá frequência 1.

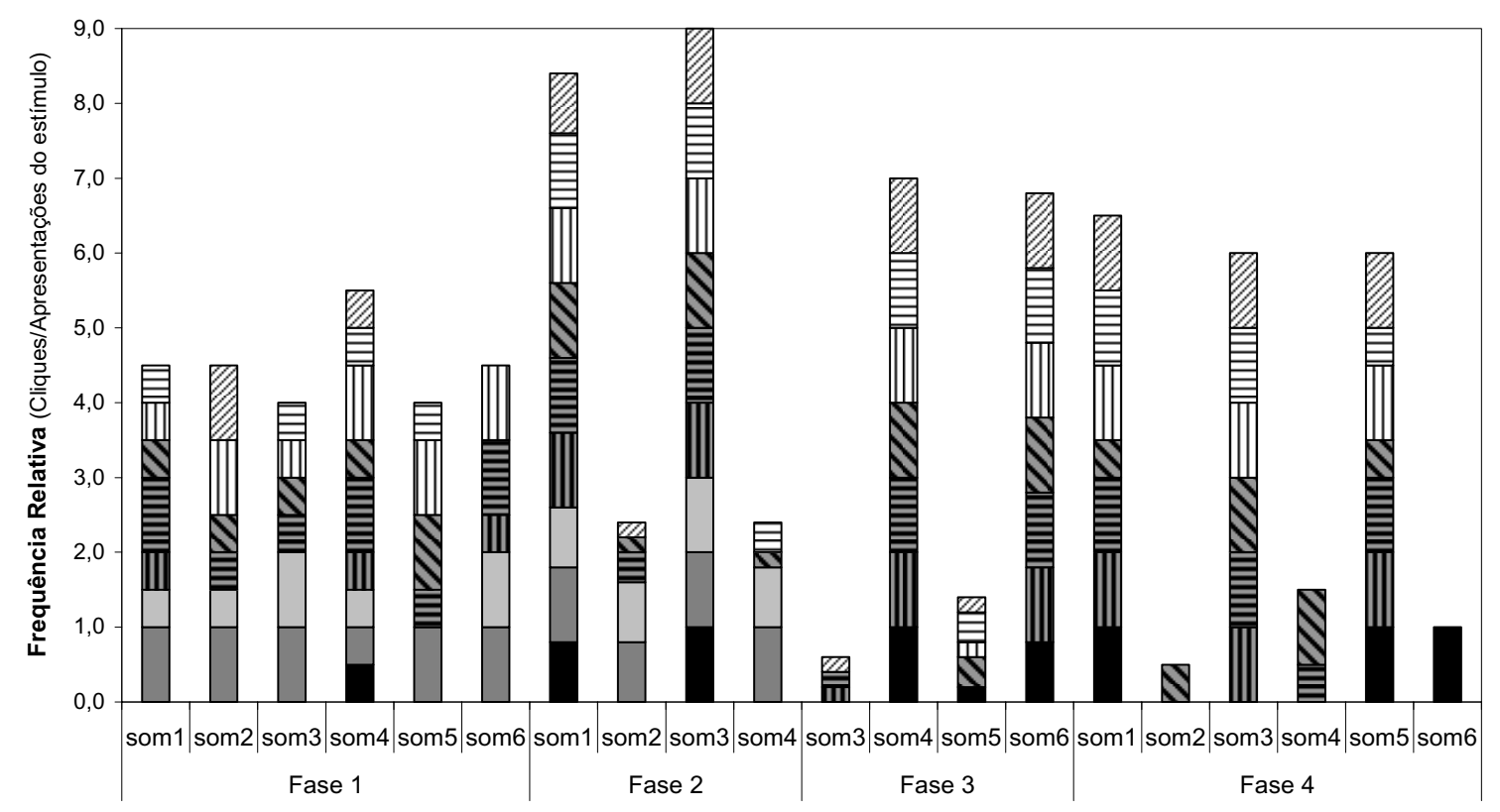

Participantes

Etapas do procedimento

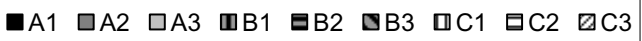

Figura 2. Freqüência relativa (resposta de clique/apresentações do estímulo) de cada participante nas condições Modo (A), Andamento (B) e Mista (C).

\section{Melodias em modo maior e Melodias em modo menor}

Esperou-se que os participantes submetidos à condição modo apresentassem dificuldades de discriminação, dada a maior complexidade desta propriedade musical. Como se percebe na Figura 2, isto foi confirmado, pois apenas um dos três participantes submetidos a essa condição conseguiu chegar à última fase do procedimento.

As medidas da linha de base (Fase 1) mostram, por um lado, que os participantes A2 e A3 clicaram para praticamente todos os estímulos apresentados. Por outro lado, A1 clicou apenas uma vez diante do som 4. Isto pode indicar que, antes da introdução do reforçamento, os participantes não discriminavam os sons apresentados clicando no botão de escolha para apenas determinado grupo.

Na Fase 2, quando o reforçamento foi inserido, houve altas taxas de respostas $(1-0,8$ respostas por apresentação de estímulo) no desempenho de A2 e A3 diante dos sons. Porém, as respostas foram emitidas indiscriminadamente. Como era de se esperar como consequência de um desempenho em extinção, A2 e A3 decidiram encerrar o procedimento após 15 e 22 repetições de fase mal-sucedidas, respectivamente.

$\mathrm{O}$ participante $\mathrm{A} 1$, a julgar pelas taxas apresentadas nas Fases 2 e 3 de treino discriminativo, discriminou os grupos de sons enquanto cada resposta correta era consequenciada com um reforçador. Contudo, em nenhuma das fases apresentou um índice discriminativo de 100\%. Consequentemente, observa-se que, na fase de teste (Fase 4), a classe de estímulos por ele formada contém dois sons em tom maior (1 e 5) e um som em tom menor (6). Esse desempenho paradoxal parece refutar a afirmação de que esse participante tenha formado classes funcionais em razão de som6 ter uma propriedade tonal diferente de 1 e 5 . Entretanto, vale lembrar que a relação entre 1 e 5 em função da melodia em tom maior não foi treinada anteriormente no procedimento, configurando-se, portanto, como uma relação emergente.

O critério estabelecido por Vaughan (1988) para a formação de classes funcionais pelo desempenho de seus pombos foi esse desempenho ocorrer de acordo com o especificado pelo SD. Entretanto, nas várias reversões discriminativas realizadas durante o seu experimento, era permitido (e também necessário) que o sujeito experimental "errasse" as primeiras apresentações de um novo bloco de figuras, discriminando a mudança da contingência, e adequando-se ao novo contexto que a mudança gerava.

Contudo, como explicar o aparecimento do som6 nessa classe controlando o desempenho de A1? Nessa condição experimental, todos os estímulos têm o mesmo andamento, o que sinalizaria a possibilidade de que A1 tenha ficado sob controle das três notas musicais compartilhadas entre os sons 1, 5 e 6. De fato, faltou a ele discriminar duas notas contidas no som 6 , diferentes das notas das melodias tocadas em 1 e 5 . Contudo, 
corroborando estudos anteriores (Bella e cols., 2001; Hyde \& Peretz, 2004), trata-se de uma tarefa difícil para indivíduos sem treino formal musical.

Em síntese, os dados da condição modo permitem duas afirmativas: (a) os resultados confirmam os encontrados em outros estudos, de que a aprendizagem do repertório de discriminar estímulos melodicamente distintos é complexa $(\mathrm{Na}-$ wrot, 2003; Bella e cols., 2001) exigindo um período mais longo de treino formal; entretanto, (b) o treino discriminativo sistemático pode ser eficiente no desenvolvimento desta habilidade.

\section{Melodias em andamento rápido e Melodias em andamento lento}

$\mathrm{Na}$ condição Andamento, era esperado que os participantes alcançassem a última fase do procedimento e que, nela, formassem classes funcionais, dado que o andamento configura-se como uma propriedade sonora mais facilmente perceptível. A despeito disto, o participante B3 não formou classes, pois emitiu a resposta de clique no botão de escolha para 5 dos 6 estímulos apresentados na Fase 4.

Esse desempenho foi uma contradição diante do fato de que esse participante repetiu as Fases 2 e 3 por três vezes cada, passando por elas com índice discriminativo de $90 \%$. Seu repertório assemelhou-se ao dos participantes submetidos ao piloto deste estudo, comportando-se, nesta fase, como nas fases anteriores: clicavam e aguardavam a confirmação do acerto (reforço). Tal jorro de respostas, padrão comum e esperado para o início de contingências de extinção (Skinner, 1953, p. 150), provavelmente controlado pela operação estabelecedora de privação do reforço (pontos), sugere que as alterações no layout das instruções da Fase 4 foram insuficientes para controlar o comportamento de todos os participantes.

Por sua vez, os participantes B1 e B2 chegaram à última fase com um desempenho demonstrativo da formação de classes de estímulos dos andamentos. O participante B1 atingiu o índice discriminativo de $100 \%$ na Fase 2 e na fase de teste. Ele clicou apenas diante de estímulos de andamento rápido (som1, som3 e som5), formando classes funcionais de andamentos.

O desempenho do participante B2 também sugere a formação de classes, embora ele tenha clicado uma vez diante de um estímulo do grupo negativo (S-). Ainda assim, embasado no trabalho de Vaughan (1988), este único erro não invalida a formação de classes funcionais, dada a necessidade da ocorrência do erro para que o sujeito discrimine a transferência da função dos estímulos.

\section{Melodias em modo maior e andamento rápido e Melodias em modo menor e andamento lento}

Indubitavelmente, esperava-se que os melhores desempenhos ocorressem nessa condição experimental, pois as configurações convergentes (modo maior e andamento rápido; modo menor e andamento lento) seriam mais facilmente discrimináveis (Gagnon \& Peretz, 2003). De fato, o grupo de participantes dessa condição foi o único que clicou apenas para estímulos de uma mesma classe na Fase 4 (C1 e C3 obtiveram índices discriminativos de $100 \%$ e C2 de 92\%), embora tenham apresentado desempenhos distintos ao longo do treino discriminativo.

A exposição a estímulos com mais propriedades (variáveis de controle) pode ter tornado a aprendizagem mais difícil, uma vez que nas Fases 2 e 3 apenas o participante C1 apresentou índice discriminativo de 100\%. Uma explicação desse fenômeno seria que, para alocar os estímulos Dó maior andamento rápido e Mi menor andamento lento em classes distintas, o participante ficou exposto a diferenças de tonalidade (Dó-Mi), de modo (Maior-Menor) e andamento (rápido-lento). Somente a combinação correta permitiria o acerto. Se, num primeiro momento, o participante ficasse sob controle do andamento e, na próxima apresentação, ficasse sob controle da tonalidade, provavelmente não alcançaria a pontuação mínima para passar de fase. Entretanto, conforme citado anteriormente, os resultados indicam que, uma vez aprendida a discriminação em função da combinação das variáveis, tal repertório tende a se manter, mesmo após a retirada do reforçador (Fase 4).

Seguindo sugestões de Sidman e cols. (1989) treinaramse as relações $\mathrm{AB}, \mathrm{BC}$, seguidas da investigação da emergência da relação AC. De fato, é justamente a combinação do treino $\mathrm{AB}$ - $\mathrm{BC}$ com a reversão discriminativa que coloca a presente pesquisa entre os trabalhos que discutem interfaces entre classes equivalentes e funcionais. Isto reforça a grande importância da Fase 4 para a demonstração da formação de classes funcionais. Somente a emergência de repertórios não treinados, de resposta comum, diante de um grupo de estímulos, demonstraria a formação das classes funcionais. A Figura 3 apresenta um comparativo dos desempenhos dos participantes que alcançaram a Fase 4.

As classes funcionais (sons 1, 3 e 5; e sons 2, 4 e 6) foram formadas na Fase 4, sobretudo pelos participantes da condição Mista (C1, C2 e C3). Fato interessante é que, para todos os participantes, a classe formada pelos sons 1,3 e 5 adquiriu função de $\mathrm{S}+$. Na fase anterior, os estímulos 3 e 5 tinham função S-, o que sugere que os sujeitos podem ter ficado sob controle da contingência que poderia ser especificada na regra mudança de fase = mudança de função, que passou a atuar a partir da mudança da reversão discriminativa da Fase 2 para a 3. Entretanto, não era o objetivo deste estudo descrever esse controle instrucional.

$\mathrm{Na}$ condição andamento, os participantes B1 e B2 formaram classes com os sons 1, 3 e 5. Entretanto, B2 incluiu, com taxa de 0,5 respostas por apresentação, o estímulo som4. É possível que erros desta natureza, a exemplo do que ocorreu para o participante B3 com os sons 2 e 4, sejam controlados por discriminações imprecisas sobre qual propriedade deveria atuar no controle da formação da classe. B3, por exemplo, clicou para todos os estímulos, exceto o som6, que é a melodia mais alta apresentada em andamento lento. 


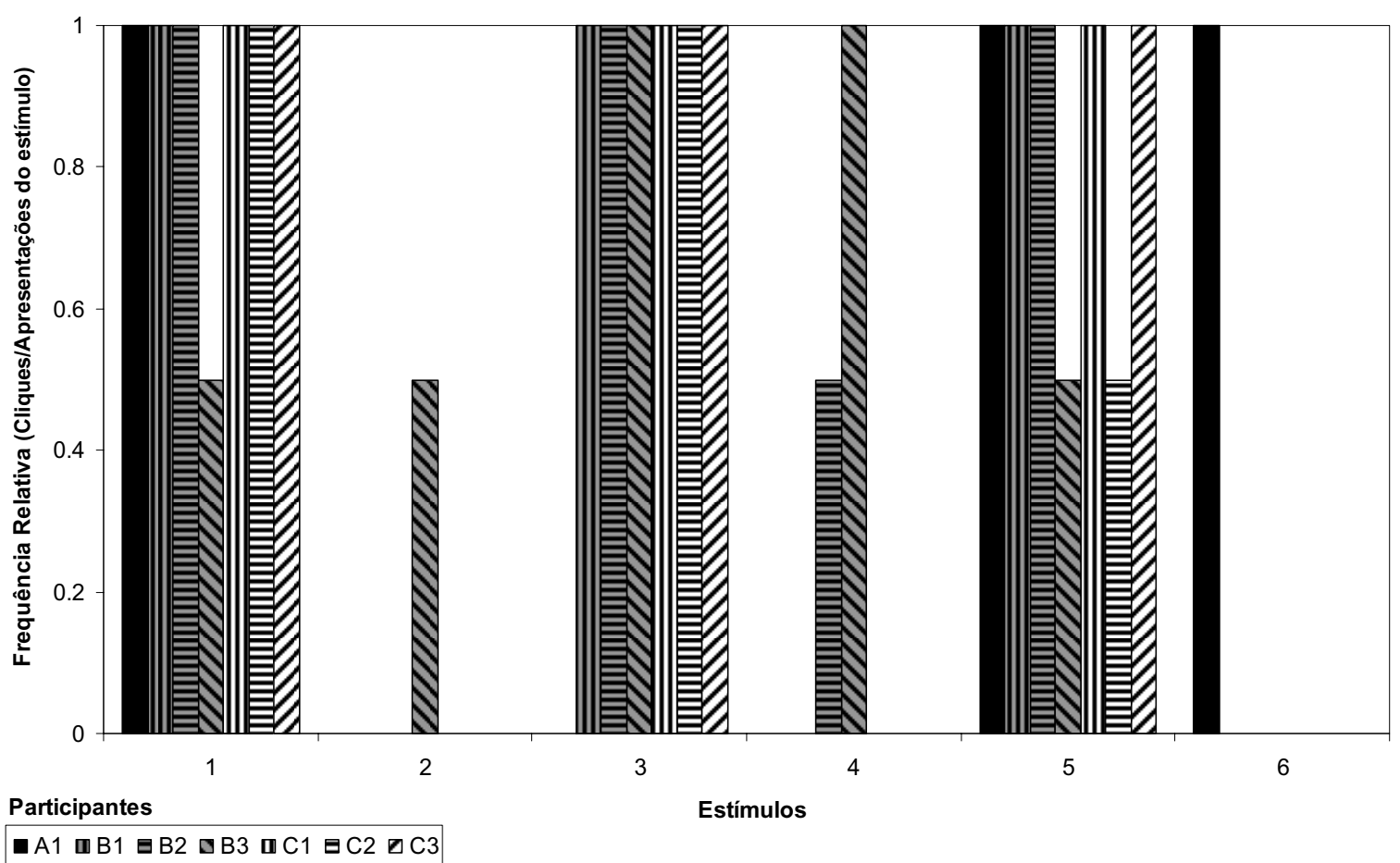

Figura 3. Comparativo do desempenho dos participantes na Fase 4.

\section{Conclusão}

Uma abordagem comportamental da música prioriza definições funcionais de eventos (públicos ou privados) observáveis (pelo pesquisador ou pelo músico; por ouvintes ou aprendizes de música) considerando sua função com outros comportamentos e estímulos do contexto analisado.

O efeito do treino discriminativo é controlar a formação de classes funcionais de estímulos sonoros. O treino proposto mostrou-se eficiente para a formação das classes funcionais de estímulos musicais. Mesmo diante das diferenças nos desempenhos dos participantes nas condições experimentais, os dados corroboraram os achados em pesquisas levantadas na revisão do tema, a saber: agrupamentos de estímulos pela propriedade andamento foram mais fáceis de discriminar do que aqueles agrupados pela melodia (Bella e cols., 2001; Hyde \& Peretz, 2004).

Além disso, a manipulação combinada das duas propriedades, em condição convergente, permitiu desempenho ainda superior dos participantes, como demonstrado na condição Mista.

Os participantes desta pesquisa não haviam sido previamente expostos a treinos formais de música. Entretanto, ao final do procedimento, que teve duração média de 10 minutos, melhoraram consideravelmente seu repertório de discriminação de sons. Se o período de treino discriminativo infinitamente curto ao qual formam submetidos for comparado a toda uma história de aprendizagem formal de música geralmente longa, a relação entre o tempo de treino e o índice discriminativo na habilidade aprendida foi provavelmente mais significativa do que seria se os participantes aprendessem tal habilidade em seu ambiente natural, sem aulas formais de música. Desta forma, uma sugestão para estudos futuros seria a aplicação do software desta pesquisa para acelerar o processo de aquisição de repertório de discriminação de propriedades de estímulos musicais.

Quanto à discussão de formação de classes funcionais, caberiam aqui sugestões de procedimento para experimentos futuros: aplicar o matching to sample com estímulos sonoros; comparar desempenhos de sujeitos submetidos ao matching to sample e ao treino discriminativo; ou mesmo combiná-los, conforme proposto por Tyndall e cols. (2004). Paralelo a isto, os participantes poderiam ser questionados sobre os critérios que utilizaram para fazer suas escolhas, a fim de investigar prováveis controles instrucionais sobre seus desempenhos.

Por fim, estudar música como analista do comportamento evoca muitas questões. Segundo Parrott (1984), as atividades envolvidas na mediação reforçadora para o comportamento do músico são apenas fases da ação do ouvinte. Do ponto de vista do processo de discriminação das propriedades musicais, o comportamento do ouvinte pode ser analisado como ouvir e compreender, fases talvez mais importantes da mediação de um ouvinte da música. Aliás, parafraseando Skinner (1957), é isto o que diferencia o comportamento verbal (vocalizado, escrito e gestualizado, pois são os mais considerados como verbais) do comportamento social geral: "O ouvinte deve responder do modo como foi precisamente condicionado com o intuito de reforçar o comportamento do (músico)" (p. 225). Dessa maneira, ouvir 
é uma relação funcional entre a resposta do ouvinte e a estimulação musical, garantida por treino especial numa cultura musical. O compreender depende do ouvir e o extrapola, no sentido em que há respostas encobertas implícitas envolvidas na reação do ouvinte (Parrott, 1984). Isto pode se dar de um modo especial na música.

Catania (1998) enfatiza que o comportamento verbal ouvido ou falado sempre envolve "palavras", independente da modalidade (falada, escrita e gestualizada, por exemplo). As unidades que funcionam como "palavras" são determinadas pelas práticas da comunidade. É possível transpor isto para a música: notas, melodias, tons, partituras, dentre outros funcionam como "palavras". Música poderia ser considerada como mais uma modalidade de "palavra" humana (ouvida, tocada), pois serve à comunicação e é uma atividade simbólica. Portanto, a arte musical seria uma forma especializada de comportamento verbal não vocalizado, e o significado dessas "palavras" está nas contingências naturais e/ou sociais que ocasionam o "fazer" música, mantidas pela comunidade verbal de músicos. Entretanto, a defesa da música como comportamento verbal depende de um refinamento de várias definições analítico-comportamentais a ser deixado para um estudo futuro.

\section{Referências}

Barmann, B. C., Croyle-Barmann, C., \& Mclain, B. (1980). The use of contingent-interrupted music in the treatment of disruptive bus-riding behavior. Journal of Applied Behavior Analysis, 13, 693-698.

Beecher, M. D., \& Harrison, J. M. (1971). Rapid acquisition of an auditory localization discrimination by rats. Journal of the Experimental Analysis of Behavior, 16, 193-199.

Bella, S. D., Peretz, I., Rousseau, L., \& Gosselin, N. (2001). A developmental study of the affective value of tempo and mode in music. Cognition, 80(3), B1-B10.

Burlile, C. J., Feldman, M. L., Craig, C., \& Harrison, J. M. (1985). Control of responding by the location of sound: Role of binaural cues. Journal of the Experimental Analysis of Behavior, 43, 315-319.

Catania, C. A. (1998). The taxonomy of verbal behavior. In K. A. Lattal \& M. Perone (Eds.), Handbook of research methods in human operant behavior (pp. 405-433). New York: Plenum.

Chlan, L. (1998). Effectiveness of a music therapy intervention on relaxation and anxiety for patients receiving ventilatory assistance. Heart \& Lung, 27(3), 169-176.

Collins, D. (2005). A synthesis process model of creative thinking in music composition. Psychology of Music, 33, 193-216.

Crites, R. J., Harris, R. T., Rosenquist, H., \& Thomas, D. R. (1967). Response patterning during stimulus generalization in the rat. Journal of the Experimental Analysis of Behavior, 10, 165-168.
De Rose, J. C. (1993). Classes de estímulos: Implicações para uma análise comportamental da cognição. Psicologia: Teoria e Pesquisa, 9, 283-303.

Downey, P., \& Harrison, J. M. (1972). Control of responding by location of auditory stimuli: Role of differential and nondifferential reinforcement. Journal of the Experimental Analysis of Behavior, 18, 453-463.

Downey, P., \& Harrison, J. M. (1975). Control of responding by sound location in monkeys: Rapid acquisition in darkness. Journal of the Experimental Analysis of Behavior, 23, 265-269.

Ebie, B. D. (2004). The effects of verbal, vocally modeled, kinesthetic, and audio-visual treatment conditions on male and female middle-school vocal music students' abilities to expressively sing melodies. Psychology of Music, 32, 405-417.

Eikeseth, S., \& Smith, T. (1992). The development of functional and equivalence classes in high-functioning autistic children: The role of naming. Journal of the Experimental Analysis of Behavior, 58, 123-133.

Farris, H. E. (1967). Classical conditioning of courting behavior in the japanese quail, coturnix japonica. Journal of the Experimental Analysis of Behavior, 10, 213-217.

Ferrari, M., \& Harris, S. L. (1981). The limits and motivating potential of sensory stimuli as reinforcers for autistic children. Journal of Applied Behavior Analysis, 14, 339-343.

Gagnon, L., \& Peretz, I. (2003). Mode and tempo relative contributions to happy-sad judments in equitone melodies. Cognition and emotion, 17, 25-40.

Galizio, M., Stewart, K. L., \& Pilgrim, C. (2004). Typicality effects in contingency-shaped generalized equivalence classes. Journal of the Experimental Analysis of Behavior, 82, 253-273.

Galvão, O. F. (1993). Classes funcionais e equivalência de estímulos. Psicologia: Teoria e Pesquisa, 9, 547-554.

Gonçalves, E. P. (2005). Conversas sobre iniciação à pesquisa científica. Campinas, SP: Alínea.

Goulart, P. R. K., Galvão, O. F., \& Barros, R. S. (2003). Busca de formação de classes de estímulos via procedimento de reversões repetidas de discriminações simples combinadas em macaco-prego (Cebus apella). Interação em Psicologia, 7(1), 109-119.

Green, S. (1975). Auditory sensitivity and equal loudness in the squirrel monkey (Saimiri sciureus). Journal of the Experimental Analysis of Behavior, 23, 255-264.

Greene, B. F., Bailey, J. S., \& Barber, F. (1981). An analysis and reduction of disruptive behavior on school buses. Journal of Applied Behavior Analysis, 14, 177-192.

Hanser, S. B., \& Thompson, L. W. (1994). Effects of a music therapy strategy on depressed older adults. Journal of Gerontology, 49(6), 265-269.

Harrison, J. M. (1988). Control of responding by sounds of different quality: An evolutionary analysis. Journal of the Experimental Analysis of Behavior, 50, 521-539. 
Harrison, J. M., \& Abelson, R. M. (1959). The maintenance of behavior by the termination and onset of intense noise. Journal of the Experimental Analysis of Behavior, 2, 23-42.

Harrison, J. M., \& Briggs, R. M. (1977). Orientation and lever responding in auditory discriminations in squirrel monkeys. Journal of the Experimental Analysis of Behavior, 28, 233-241.

Harrison, J. M., Iversen, S. D., \& Pratt, S. R. (1977). Control of responding by location of auditory stimuli: Adjacency of sound and response. Journal of the Experimental Analysis of Behavior, 28, 243-251.

Hatem, T. P., Lira, P. I. C., \& Mattos, S. S. (2006). Efeito terapêutico da música em crianças em pós-operatório de cirurgia cardíaca. Jornal de Pediatria, 82, 186-192.

Hayes, L. J., Thompson, S., \& Hayes, S. C. (1989). Stimulus equivalence and rule following. Journal of the Experimental Analysis of Behavior, 52, 275-291.

Herman, L. M., \& Gordon, J. A. (1974). Auditory delayed matching in the bottle-nose dolphin. Journal of the Experimental Analysis of Behavior, 21, 19-26.

Hoskyns, S. (1988). Studying group music therapy with adult offenders: Research in progress. Psychology of Music, 16, 25-41.

Hume, K. M., \& Crossman, J. (1992). Musical reinforcement of practice behaviors among competitive swimmers. Journal of Applied Behavior Analysis, 25, 665-670.

Hyde, K. L., \& Peretz, I. (2004). Brains that are out of tune but in time. Psychological Science, 15, 356-360.

Jitsumori, M., Siemann, M., Lehr, M., \& Delius, J. D. (2002). A new approach to the formation of equivalence classes in pigeons. Journal of the Experimental Analysis of Behavior, 78, 397-408.

Kelly, D. D. (1980). Enhancement of conditioned automatic responses in monkeys when preshock signals occasion operant suppression. Journal of the Experimental Analysis of Behavior, 33, 275-284.

Kennedy, C. H., \& Souza, G. (1995). Functional analysis and treatment of eye poking. Journal of Applied Behavior Analysis, 28, 27-37.

Koger, S. M., Chapin, K., \& Brotons, M. (1999). Is music therapy an effective intervention for dementia? A metaanalytic review of literature. Journal of Music Therapy, 36, 2-15.

Lewis, P., \& Stoyak, M. (1979). Signal-controlled responding. Journal of the Experimental Analysis of Behavior, 31, 115-125.

Lowe, C. F., Beasty, A., \& Bentall, R. P. (1983). The role of verbal behavior in human learning: Infant performance on fixed-interval schedules. Journal of the Experimental Analysis of Behavior, 39, 157-164.

Lundin, R. W. (1977). Personalidade: Uma análise do comportamento. São Paulo: EPU.

Macdonald, R. A. R., Mitchell, L. A., Dillon, T., Serpell, M. G., Davies, J. B., \& Ashley, E. A. (2003). An empirical investigation of the anxiolytic and pain reducing effects of music. Psychology of Music, 31, 187-203.
Maltzman, E., Holz, W. C., \& Kunze, J. (1965). Supplementary knowledge of results. Journal of the Experimental Analysis of Behavior, 8, 385-388.

Mcadie, T. M., Foster, T. M., \& Temple, W. (1996). Concurrent schedules: Quantifying the aversiveness of noise. Journal of the Experimental Analysis of Behavior, 65, 37-55.

McPherson, G. E. (2005). From child to musician: Skill development during the beginning stages of learning an instrument. Psychology of Music, 33, 5-35.

Meehan, E. F. (1999). Class-consistent differential reinforcement and stimulus class formation in pigeons. Journal of the Experimental Analysis of Behavior, 72, 97-115.

Meltzoff, J. (2001). Critical thinking about research. Washington, DC: APA.

Muniz, E. L., \& Castro, H. M. T. (2005). Dicionário barsa da língua portuguesa. São Paulo: Barsa Planeta.

Nawrot, E. (2003). The perception of emotional expression in music: Evidence from infants, children and adults. Psychology of Music, 31, 75-92.

Neill, J. C., \& Harrison, J. M. (1987). Auditory discrimination: The Kornorski quality-location effect. Journal of the Experimental Analysis of Behavior, 48, 81-95.

Neill, J. C., Liu, Z., Mikati, M., \& Holmes, G. L. (2005). Pilocarpine seizures cause age-dependent impairment in auditory location discrimination. Journal of the Experimental Analysis of Behavior, 84, 357- 370.

Nilsson, U., Unosson, M., \& Rawal, N. (2005). Stress reduction and analgesia in patients exposed to calming music postoperatively: A randomized controlled trial. European Journal of Anesthesiology, 22, 96-102.

North, A. C., \& Hargreaves, D. J. (1999). Can music move people? The effects of musical complexity and silence on waiting time. Environment and Behavior, 31, 136-149.

North, A. C., Shilcock, A., \& Hargreaves, D. J. (2003). The effect of musical style on restaurant customers' spending. Environment and Behavior, 35, 712-718.

Odell, H. A (1988). Music therapy approach in mental health. Psychology of Music, 16, 52-61.

Parrot, L. J. (1984). Listening and understanding, The Behavior Analyst, 7, 29-39.

Pierrel, R., \& Sherman, J. G. (1960). Generalization of auditory intensity following discrimination training. Journal of the Experimental Analysis of Behavior, 3, 313-322.

Raslear, T. G. (1975). The effects of varying the distribution of generalization stimuli within a constant range upon the bisection of a sound-intensity interval by rats. Journal of the Experimental Analysis of Behavior, 23, 369-375.

Raslear, T. G., Pierrel-Sorrentino, R., \& Brissey, C. (1975). Concurrent assessment of schedule and intensity control across successive discriminations. Journal of the Experimental Analysis of Behavior, 23, 247-254.

Reed, P., Howell, P., Sackin, S., Pizzimenti, L., \& Rosen, S. (2003). Speech perception in rats: Use of duration and rise time cues in labeling of affricate/fricative sounds. Journal of the Experimental Analysis of Behavior, 80, 205-215. 
Sadakata, M., Ohgushi, K., \& Desain, P. (2004). A crosscultural comparison study of the production of simple rhythmic patterns. Psychology of Music, 32, 389-403.

Schusterman, R. J., \& Kastak, D. (1998). Functional equivalence in a California sea lion: Relevance to animal social and communicative interactions. Animal Behavior, 55, 1087-1095.

Schusterman, R. J., Balliet, R. F., \& Nixon, J. (1972). Underwater audiogram of the california sea lion by the conditioned vocalization technique. Journal of the Experimental Analysis of Behavior, 17, 339-350.

Segal, M., \& Harrison, J. M. (1978). The control of responding by auditory stimuli: Interactions between different dimensions of the stimuli. Journal of the Experimental Analysis of Behavior, 30, 97-106.

Sidman, M. (1992). Equivalence relations: Some basic considerations. In S. C. Hayes, \& L. J. Hayes (Eds.), Understanding verbal relations (pp. 15-28). Reno: Context Press.

Sidman, M., \& Tailby, W. (1982). Conditional discrimination vs. matching to sample. An expansion of the testing paradigm. Journal of the Experimental Analysis of Behavior, 43, 21-42.

Sidman, M., Wynne, C. K., Maguire, R. W., \& Barnes, T. (1989). Functional classes and equivalence relations. Journal of the Experimental Analysis of Behavior, 52, 261-274.

Skinner, B. F. (1953). Science and human behavior. New York: Macmillan.

Skinner, B. F. (1957). Verbal behavior. New York: AppletonCentury-Crofts.

Stebbins, W. C. (1966). Auditory reaction time and the derivation of equal loudness contours for the monkey. Journal of the Experimental Analysis of Behavior, 9, 135-142.

Stromer, R., Mackay, H. A., \& Remington, B. (1996). Naming, the formation of stimulus lasses, and applied behavior analysis. Journal of Applied Behavior Analysis, 29, 409-431.

Thompson, T., Heistad, G. T., \& Palermo, D. S. (1963). Effect of amount of training on rate and duration of responding during extinction. Journal of the Experimental Analysis of Behavior, 6, 155-161.

Todres, I. D. (2006). Música é remédio para o coração. Jornal de Pediatria, 82, 166-168.

Tomonaga, M. (1999). Establishing functional classes in a chimpanzee (pan troglodytes) with a two-item sequentialresponding procedure. Journal of the Experimental Analysis of Behavior, 72, 57-79.

Tyndall, I. T., Roche, B., \& James, J. E. (2004). The relation between stimulus function and equivalence class formation. Journal of the Experimental Analysis of Behavior, 81, 257-266.

Vaughan, W., Jr. (1988). Formation of equivalence sets in pigeons. Journal of Experimental Psychology: Animal Behavior Processes, 14, 36-42.
Vollmer, T. R., \& Iwata, B. A. (1991). Establishing operations and reinforcement effects. Journal of Applied Behavior Analysis, 24, 279-291.

White, J. M. (1992). Music therapy: An intervention to reduce anxiety in the myocardial infarction patient. Clinical Nurse Specialist, 6, 58-63.

Wilson, C. W., \& Hopkins, B. L. (1973). The effects of contingent music on the intensity of noise in junior high home economics classes. Journal of Applied Behavior Analysis, 6, 269-275.

Wilson, S. (2003). The effect of music on perceived atmosphere and purchase intentions in a restaurant. Psychology of Music, 31, 93-112.

Wirth, O., \& Chase, P. N. (2002). Stability of functional equivalence and stimulus equivalence: Effects of baseline reversals. Journal of the Experimental Analysis of Behavior, 77, 29-47.

Alex Roberto Machado é Professor Titular da Faculdade Pitágoras, unidade Linhares, Mestre em Psicologia pela Universidade Federal do Espírito Santo.

Elizeu Batista Borloti é Professor Adjunto do Departamento de Psicologia Social e do Desenvolvimento da Universidade Federal do Espírito Santo, Pós-doutorando pela Universidade de São Paulo.
Recebido: $13 / 05 / 2008$

$1^{a}$ revisão: $21 / 11 / 2008$

$2^{a}$ revisão: $24 / 01 / 2009$

$3^{a}$ revisão: 03/03/2009

Aceite final: 18/03/2009 